02

\title{
Избыточная проводимость анизотропных неоднородных сверхпроводников при температуре выше критической
}

\author{
() Т.И. Могилюк ${ }^{1}$, П.Д. Григорьев ${ }^{2,3,4}$, К.К. Кешарпу ${ }^{3}$, И.А. Колесников ${ }^{3}$, \\ А.А. Синченко ${ }^{5,6}$, А.В. Фролов ${ }^{6}$, А.П. Орлов ${ }^{6}$ \\ ${ }^{1}$ Научный исследовательский центр „Курчатовский институт“, \\ Москва, Россия \\ ${ }^{2}$ Институт теоретической физики им. Л.Д. Ландау РАН, \\ Черноголовка, Россия \\ ${ }^{3}$ Национальный исследовательский технологический университет „МИСиС“, \\ Москва, Россия \\ ${ }^{4}$ Физический институт им. П.Н. Лебедева РАН, \\ Москва, Россия \\ ${ }^{5}$ Московский государственный университет им. М.В. Ломоносова, \\ Москва, Россия \\ ${ }^{6}$ Институт радиотехники и электроники им. В.А. Котельникова РАН, \\ Москва, Россия \\ E-mail: 5taras@mail.ru
}

Поступила в Редакцию 15 апреля 2019 г.

В окончательной редакции 22 апреля 2019 г.

Принята к публикации 24 апреля 2019 г.

\begin{abstract}
Развита теоретическая модель проводимости слоистого анизотропного нормального металла, содержащего малые сверхпроводящие гранулы эллипсоидной формы с произвольным отношением полуосей. Проведено сравнение расчетов, выполненных в рамках двух простейших приближений: самосогласованном и максвелловском. Результаты применимы для анализа наблюдаемой температурной зависимости анизотропии проводимости в различных анизотропных сверхпроводниках, если сверхпроводящая фаза проявляется неоднородно в форме изолированных сверхпроводящих гранул. Экспериментально исследована температурная зависимость электрического сопротивления вдоль и поперек проводящих слоев выше и вблизи температуры сверхпроводящего перехода для мостиковых структур различной толщины. Показана сильная зависимость этого сопротивления и даже эффективной температуры сверхпроводящего перехода от толщины мостика, то есть от количества слоев через которые течет электрический ток. При этом существенные различия наблюдались только для сопротивления поперек слоев, тогда как вдоль слоев подобных заметных различий не обнаружено.
\end{abstract}

Ключевые слова: проводимость, сверхпроводники, анизотропия, неоднородность.

DOI: $10.21883 /$ FTT.2019.09.48096.26N

\section{1. Введение}

Повышение температуры сверхпроводящего перехода $T_{c}$ в сверхпроводящих материалах является одной из основных задач в физике конденсированных сред. Большинство высокотемпературных сверхпроводников (ВТСП) обыкновенно представляют собой металлические сплавы или сложные оксидные керамики. Оксиды меди [1-4] и сверхпроводники на основе железа [5-7] представляют особый интерес, потому что $T_{c}$ в этих материалах является самой высокой при атмосферном давлении и может контролироваться в некотором диапазоне химическим составом и уровнем легирования. Во время процесса легирования легирующая добавка не осаждается равномерно, что превращает исходное пространственно однородное соединение в гетерогенный материал. Из-за этой неоднородности сверхпроводящие свойства часто наблюдаются в первую очередь в пространственно несвязанных частях материалов при
$T>T_{c}$, что является одним из возможных сценариев образования псевдощели в купратных ВТСП [8]. Когда температура $T$ приближается к $T_{c}$, небольшие сверхпроводящие области становятся больше и их фазы становятся когерентными. Наконец при $T=T_{c}$ весь объем становится сверхпроводящим. Этот тип неоднородного развития сверхпроводящих свойств выше $T_{c}$ был назван Gossamer Superproductivity [9]. Он также может называться гетерогенной сверхпроводимостью. Существование такой гетерогенной фазы было подтверждено экспериментами по магнитному отклику с использованием метода сканирующей SQUID микроскопии (scan SQUID microscopy) [10]. Пространственная неоднородность в сверхпроводнике может увеличить $T_{c}$, как показано теоретически в работах $[5,11]$. Явление также ответственно за сверхпроводящие эффекты выше $T_{c}$. Понимание связи между пространственной неоднородностью и высокотемпературной сверхпроводимостью очень важно для синтеза новых материалов с более высоким $T_{c}$. 
Недавно было высказано предположение, что избыточная проводимость слоистого анизотропного гетерогенного соединения, обусловленная изолированными сверхпроводящими островками, также является очень анизотропной с максимальным эффектом вдоль наименее проводящего направления $[9,12]$. Количественное описание этого эффекта с использованием приближения Максвелла-Гарнетта было предложено и использовано для анализа экспериментальных данных по проводимости и диамагнитному отклику в FeSe $[9,12]$. В работе [13] проводилось сравнение результатов по расчетам проводимости в рамках самосогласованного (см. краткий обзор в $[14,15])$ и максвелловского приближений для сфероидных сверхпроводящих гранул. В другой работе [16] применялось максвелловское приближение в анизотропной системе с эллипсоидными сверхпроводящими гранулами включений.

$\mathrm{FeSe}$ имеет самый простой химический состав среди большого семейства высокотемпературных сверхпроводников на основе железа, но его электронная структура и свойства очень интересны. Под давлением температура его перехода повышается до $40 \mathrm{~K}$ [17]. Очень многообещающим является наблюдение сверхпроводимости с $T_{c}>100 \mathrm{~K}$ в монослое $\mathrm{FeSe}$ на подложке $\mathrm{SrTiO}_{3}$ [18]. Объемный $\mathrm{FeSe}$ имеет неоднородную микроскопическую структуру и $T_{c} \approx 8 \mathrm{~K}[19]$. Это делает FeSe очень удобным для сравнения с нашей моделью анизотропного гетерогенного проводника. Такое сравнение с результатами расчета в максвелловском приближении в предположении сфероидной формы сверхпроводящих включений было проведено в статье [12].

\section{2. Эксперимент}

В этой работе мы провели дополнительное экспериментальное исследование температурной зависимости сопротивления для разных толщин образцов как поперек слоев, так и вдоль. При этом существенная зависимость от толщины наблюдалась только для сопротивления поперек слоев. Поэтому в качестве нового экспериментального результата мы отмечаем, что наблюдаемая анизотропия избыточной проводимости выше $T_{c}$ сильно зависит от толщины образца (мостика). На рисунке показано, как можно менять температуру сверхпроводящего FeSe, меняя толщину мезоструктуры или мостика, вырезанного в монокристалле FeSe.

Верхняя черная кривая на рисунке соответствует толщине образца $300 \mathrm{~nm}$. Для остальных кривых в процессе травления этот параметр не определялся - это достаточно сложно сделать для коротких перекрытий. Но латеральная геометрия для всех образцов одна и та же $2 \times 2 \mu \mathrm{m}$. Следовательно, уменьшение сопротивления пропорционально уменьшению толщины образца. Отсюда для нижней синей кривой получаем толщину образца 40-60 nm, что все равно много больше постоянной решетки FeSe. Удивительно, что чем меньше толщина

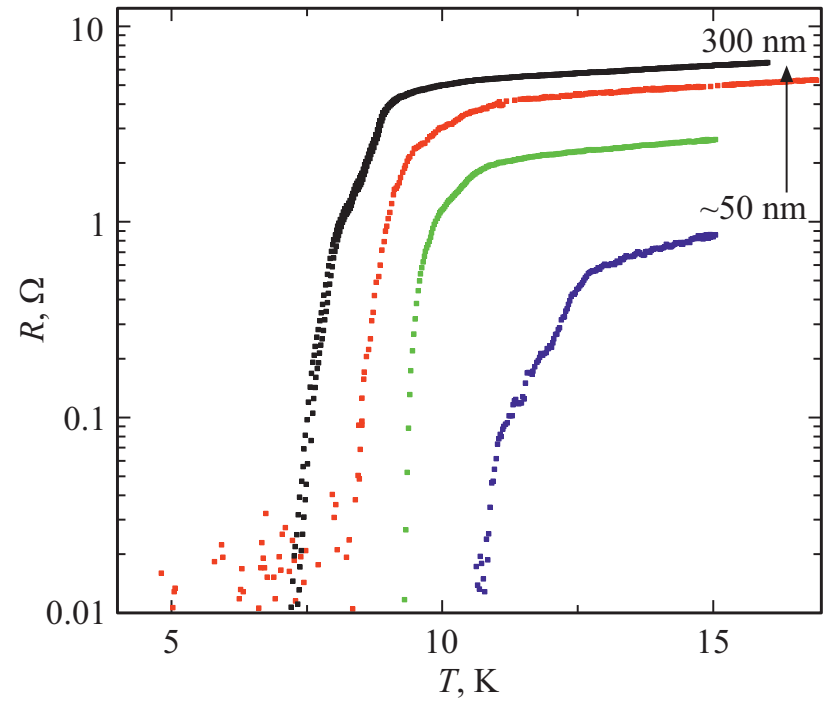

Температурная зависимость электрического сопротивления $R(T)$ и наблюдаемые по ней сверхпроводящие переходы в $\mathrm{FeSe}$ для структур с разным числом слоев. Поперечные размеры одинаковы $-2 \times 2 \mu \mathrm{m}$. Сопротивление прямо пропорционально толщине структуры, а толщина пропорциональна числу слоев. Верхняя черная кривая соответствует толщине $300 \mathrm{~nm}$, а нижняя синяя $-40-60 \mathrm{~nm}$.

(сопротивление), тем выше наблюдаемая температура сверхпроводящего перехода. При этом существенная зависимость от толщины наблюдалась только для сопротивления поперек слоев, что оказывается еще более удивительным. Заметим, что в рамках масвелловского приближения, даже при зависящей от толщины доли сверхпроводящей фазы, такой результат не получается.

\section{3. Теория}

В данной работе используется уже самосогласованное приближение с эллипсоидной формой сверхпроводящих включений. Теоретическая модель, предложенная в работе, в пределе малой концентрации сверхпроводящей фазы совпадает с максвелловским приближением и имеет простую физическую иллюстрацию $[9,12]$. Согласно этой иллюстрации, два канала межслоевого транспорта существуют в анизотропном проводнике, содержащем изолированные сверхпроводящие включения с малой объемной долей. Первый канал - ток, текущий строго в направлении электрического поля (через слои). Второй канал тока течет от одного сверхпроводящего включения к другому вдоль проводящих слоев, протекая от одного слоя к соседнему только по сверхпроводящим включениям и минуя несверхпроводящую фазу.

Рассмотрим однородную анизотропную проводящую среду с проводимостями $\sigma_{x x}^{m}, \sigma_{y y}^{m}, \sigma_{z z}^{m}$. Уравнение для непрерывности электрического тока записывается как

$$
-\nabla \mathbf{j}=\sigma_{x x}^{m} \frac{\partial^{2} V}{\partial x^{2}}+\sigma_{y y}^{m} \frac{\partial^{2} V}{\partial y^{2}}+\sigma_{z z}^{m} \frac{\partial^{2} V}{\partial z^{2}}=0 .
$$


Величина $\mathbf{j}$ - плотность тока, $V$ - электростатический потенциал. Замена координат:

$$
x=x^{\prime}, \quad y=\sqrt{\mu} y^{\prime}, \quad z=\sqrt{\eta} z^{\prime},
$$

где

$$
\mu=\frac{\sigma_{y y}^{m}}{\sigma_{x x}^{m}}, \quad \eta=\frac{\sigma_{z z}^{m}}{\sigma_{x x}^{m}},
$$

переводит (1) в уравнение Лапласа

$$
\sigma_{x x}^{m}\left(\frac{\partial^{2} V}{\partial x^{\prime 2}}+\frac{\partial^{2} V}{\partial y^{\prime 2}}+\frac{\partial^{2} V}{\partial z^{\prime 2}}\right)=0 .
$$

Если включения не сферические, а имеют эллипсоидные форму с полуосями $a, b=\beta a$ и $c=\gamma a$ и главными осями вдоль координатных осей, то после преобразования координат к изотропной среде полуоси эллипсоидов включений изменятся на

$$
a_{x}, a_{y}=a_{x} \beta / \sqrt{\mu}, \quad a_{z}=a_{x} \gamma / \sqrt{\eta} .
$$

Применяем уравнение (18.19) из книги [15] для нахождения эффективной проводимости $\sigma_{e}$ в рамках самосогласованного приближения эффективной среды для изотропной системы

$$
\left.\left.\phi_{s} \boldsymbol{\sigma}_{s}-\boldsymbol{\sigma}_{e}\right) R^{s e}+\phi_{m} \boldsymbol{\sigma}_{m}-\boldsymbol{\sigma}_{e}\right) R^{m e}=0,
$$

где $R^{l e}=\left[I+A^{*} \boldsymbol{\sigma}_{e}^{-1}\left(\boldsymbol{\sigma}_{l}-\boldsymbol{\sigma}_{e}\right)\right]^{-1}, I-$ единичная матрица, $\sigma_{l}=I \sigma_{l}(l=\{s, m\}), \sigma_{s, m}$ - проводимость сверхпроводящей $(s)$ и металлической фаз $(m)\left(\sigma_{m} \equiv \sigma_{x x}^{m}\right)$, $\phi_{s, m}$ - объемная доля сверхпроводящей $(s)$ и металлической фаз $(m)$, а эффективные тензоры проводимости $\boldsymbol{\sigma}_{e}$ и деполяризации $A^{*}$ диагональны:

$$
\boldsymbol{\sigma}_{e}=\left[\begin{array}{ccc}
\sigma_{x x} & 0 & 0 \\
0 & \sigma_{y y} & 0 \\
0 & 0 & \sigma_{z z}
\end{array}\right], \quad \mathbf{A}^{*}=\left[\begin{array}{ccc}
A_{x} & 0 & 0 \\
0 & A_{y} & 0 \\
0 & 0 & A_{z}
\end{array}\right] .
$$

Тензор деполяризации эллипсоида (см. формулу (6) в работе [20] или секцию 17.1.2 из книги [15] (по нашему убеждению, в формуле (17.25) коэффициент 2 входит в степени 1 , а не $j$, иначе не выполняется условие (17.26) на равенство следа тензора 1)) имеет главные значения

$$
A_{i}=\frac{a_{x} a_{y} a_{z}}{2} \int_{0}^{+\infty} \frac{d t}{\left(t+a_{i}^{2}\right) \sqrt{\left(t+a_{x}^{2}\right)\left(t+a_{y}^{2}\right)\left(t+a_{z}^{2}\right)}} .
$$

Интегралы (8) были вычислены аналитически в работе [16]. Подставляя значения $\sigma_{m}, A^{*}, \sigma_{e}$ и $\phi_{m}=\left(1-\phi_{s}\right)$ в уравнение (6) и упрощая с использованием обстоятельства, что проводимость сверхпроводящих гранул $\sigma_{s} \rightarrow \infty$ бесконечна, находим выражение для тензора проводимости

$$
\frac{\sigma_{i i}}{\sigma_{m}}=\frac{1}{1-\phi_{s} / A_{i}} .
$$

Возвращаясь от координат изотропной среды к исходным координатам анизотропной, выводим окончательное выражение для проводимости вдоль трех главных осей неоднородной анизотропной среды с эллипсоидными сверхпроводящими включениями

$$
\frac{\sigma_{i i}}{\sigma_{i i}^{m}}=\frac{1}{1-\phi_{s} / A_{i}} .
$$

Полученные формулы содержат неявную зависимость от температуры через температурную зависимость доли сверхпроводящей фаз $\phi_{s}$. Она может быть получена из данных по диамагнитному отклику, например, как это сделано в работах $[9,12,16]$. На рис. 3,4 в статье [12] для $\mathrm{FeSe}$ и на рис. 3 в статье [16] для органического сверхпроводника $\beta$-(BEDT-TTF) ${ }_{2} \mathrm{I}_{3}$ показана такая зависимость $\phi_{s}(T)$; она похожа на степенную в широком диапазоне температур $T>T_{c}$ и резко обрывается в ноль при $T \sim 5 T_{c}$ (см. рис. 4, $d$ в статье [9]). Заметим, что эти зависимости $\phi_{s}(T)$ для $\mathrm{FeSe}$ и для $\beta$-(BEDT-TTF $)_{2} \mathrm{I}_{3}$, хоть и качественно похожи, различаются количественно, поскольку функция $\phi_{s}(T)$ определяется свойствами и природой беспорядка, приводящего к пространственной неоднородности, который зависит от вещества и даже от образца. Если данные по диамагнитному отклику не доступны, зависимость $\phi_{s}(T)$ может быть определена согласно формулам (10) или (11) из температурной зависимости избыточной проводимости вдоль одной оси, например $z$, а температурная зависимость вдоль двух других осей находится из полученных формул и позволяет сравнить результаты расчета в рамках этой модели с экспериментальными данными (см. рис. 2 в статье [16]).

\section{4. Обсуждение}

Выше выведено аналитическое выражение (10) в рамках классической модели для проводимости в анизотропных материалах вблизи температуры сверхпроводящего перехода с изолированными островками сверхпроводящих включений. Сверхпроводящие гранулы имели форму эллипсоида и были ориентированы в пространстве одинаково. Область применимости классической модели подробно обсуждалась [16] и предполагает, что размер гранул много больше толщины когерентности сверхпроводника или что эффекты близости сверхпроводимости не сильно меняют форму эффективных сверхпроводящих островков.

Выведенное уравнение (10), полученное в рамках самосогласованного приближения, сильно отличается от выражения

$$
\frac{\sigma_{i i}^{*}(\phi)}{\sigma_{i i}^{m}}=1+\frac{\phi_{s}}{A_{i}\left(1-\phi_{s}\right)},
$$

полученного [16] в рамках максвелловского приближения, хотя эти две формулы и совпадают в пределе $\phi_{s} \ll A_{i}<1^{1}$. Заметим, что каждое из двух прибли-

\footnotetext{
${ }^{1} A_{i}<1$ всегда, поскольку $\sum_{i} A_{i}=1$ и $A_{i}>0$ [19]. Поэтому условие $\phi_{s} \ll A_{i}$ является более строгим, чем условие $\phi_{s} \ll 1$ применимости максвелловского приближения.
} 
жений, максвелловское и самосогласованное, является точным для определенного типа пространственной неоднородности [15]. Имеется несколько важных отличий этих приближений [15]. Самым главным, на наш взгляд, является порог перколяции $\phi_{s}^{c}$, когда проводимость обращается в бесконечность. Для максвелловского приближения порог перколяции одинаковый вдоль всех направлений и равен $\phi_{s}^{c}=1$. Напротив, в самосогласованном приближении $\phi_{s}^{c}=A_{i}$ различен вдоль разных направлений, будучи намного меньше вдоль оси наименьшей проводимости. Сама возможность такого анизотропного порога перколяции в бесконечном образце является дискуссионной, однако ряд экспериментов [21] указывают на подобное явление в органическом металле (TMTSF $)_{2} \mathrm{PF}_{6}$. В образцах конечного размера анизотропный порог перколяции (сверхпроводящий переход) возможен, например, если размер сверхпроводящих островков сравним в размером образца в одном направлении.

Следует отметить, что выведенные формулы (10) для проводимости в анизотропном неоднородном проводнике в самосогласованном приближении, как и полученные ранее формулы (11) в максвелловском приближении, напрямую не объясняют наблюдаемую зависимость эффективной температуры сверхпроводящего перехода от толщины образца. Однако в них сверхпроводящий переход (порог перколяции), определяющий температуру перехода $T_{c}$, вдоль одного направления может смещаться относительно $T_{c}$ вдоль другого направления, и зависит от формы сверхпроводящих включений. Поэтому если предположить, что форма сверхпроводящих островков изменяется в зависимости от толщины мезоструктуры, то можно предложить возможное объяснение наблюдаемому эффекту. Наблюдаемую зависимость эффективной температуры сверхпроводящего перехода от толщины образца в рамкам рассматриваемого самосогласованного приближения можно также объяснить предположив, что из-за поверхностных эффектов объемная доля сверхпроводящей фазы выше в более тонком образце. Однако это предположение также требует микроскопического обоснования.

\section{Финансирование работы}

Т.И.М. благодарит за финансовую поддержку РФФИ (гранты 18-02-01022, 18-02-00280, 18-32-00205, 1902-01000). П. Д. Г. благодарит госзадание 0033-20190001 „Развитие теории конденсированного состояния вещества“. Работа частично поддержана фондом развития теоретической физики и математики „Базис“. А. А. С. благодарен за поддержку РФФИ (№ 17-2910007). А. В. Ф. и А. П. О. выполняли работу в рамках государственного задания ИРЭ РАН.

\section{Конфликт интересов}

Авторы заявляют, что у них нет конфликта интересов.

\section{Список литературы}

[1] K. Jung. Met. Mater. 2, 219 (1996).

[2] G. Blumberg, M. Kang, M. V. Klein, K. Kadowaki, C. Kendziora. Science 278, 1427 (1997).

[3] K.A.Müller. J. Phys.: Condens. Matter 19, 251002 (2007).

[4] B. Keimer, S.A. Kivelson, M.R. Norman, R. Uchida, J. Zaanen. Nature 518, 965 (2015).

[5] M. Rotter, M. Pangerl, M. Tegel, D. Johrendt. Angew. Chem. 47, 7949 (2008).

[6] J.-H. Chu, J.G. Analytis, C. Kucharczyk, I.R. Fisher. Phys. Rev. B 79, 014506 (2009).

[7] Q. Si, R. Yu, E. Abrahams. Nature Rev. Mater. 1, 16017 (2016).

[8] V.Z. Kresin, Y.N. Ovchinnikov, S.A. Wolf. Phys. Rep. 431, 231 (2006).

[9] A.A. Sinchenko, P.D. Grigoriev, A.P. Orlov, A.V. Frolov, A. Shakin, D.A. Chareev, O.S. Volkova, A.N. Vasiliev. Phys. Rev. B 95, 165120 (2017).

[10] I.Iguchi, T. Yamaguchi, A. Sugimoto. Nature 412, 420 (2001).

[11] I. Martin, D. Podolsky, S.A. Kivelson. Phys. Rev. B 72, 060502 (2005).

[12] P.D. Grigoriev, A.A. Sinchenko, K.K. Kesharpu, A. Shakin, T.I. Mogilyuk, A.P. Orlov, A.V. Frolov, D.S. Lyubshin, D.A. Chareev, O.S. Volkova, A.N. Vasiliev. JETP Lett. 105, 786 (2017).

[13] K.K. Kesharpu, P.D. Grigoriev, D.I. Lazeva, T.I. Mogilyuk. Submitted J. Phys.: Conf. Ser.; arXiv:1807.01887.

[14] R. Landauer. AIP Conf. Proc. 40, 2 (1978).

[15] S. Torquato. Random heterogeneous materials: microstructure and macroscopic properties. Springer-Verlag, N.Y. (2002).

[16] S.S. Seidov, K.K. Kesharpu, P.I. Karpov, P.D. Grigoriev. Phys. Rev. B 98, 014515 (2018).

[17] S. Medvedev, T.M. McQueen, I.A. Troyan, T. Palasyuk, M.I. Eremets, R.J. Cava, S. Naghavi, F. Casper, V. Ksenofontov, G. Wortmann, C. Felser. Nature Mater. 8, 630 (2009).

[18] J.F. Ge, Z.L. Liu, C. Liu, C.L. Gao, D. Qian, Q.K. Xue, Y. Liu, J.F. Jia. Nature Mater. 14, 285 (2015).

[19] Yu.G. Naidyuk, G. Fuchs, D.A. Chareev, A.N. Vasiliev. Phys. Rev. B 93, 144515 (2016).

[20] S. Giordano. J. Electrostat. 58, 59 (2003).

[21] N. Kang, B. Salameh, P. Auban-Senzier, D. Jerome, C.R. Pasquier, S. Brazovskii. Phys. Rev. B 81, 100509 (R), (2010).

Редактор Т.Н. Василевскал 